\title{
Decision Making in Interventional Oncology: Intra-arterial Therapies for Metastatic Colorectal Cancer-Y90 and Chemoembolization
}

\author{
Alexandra H. Fairchild, MD ${ }^{1}$ Sarah B. White, MD, MS ${ }^{1}$ \\ ${ }^{1}$ Division of Vascular and Interventional Radiology, Department of \\ Radiology, Medical College of Wisconsin, Milwaukee, Wisconsin \\ Address for correspondence Sarah B. White, MD, MS, Division of \\ Vascular and Interventional Radiology, Department of Radiology, \\ Medical College of Wisconsin, 9200 W. Wisconsin Avenue, Milwaukee, \\ Semin Intervent Radiol 2017;34:87-91. \\ WI 53226 (e-mail: sbwhite@mcw.edu).
}
Abstract
Keywords
- interventional radiology
- cTACE
- DEBIRI
- radioembolization
- colorectal liver metastases

Colorectal cancer is the third most common cancer in the United States and the liver is the most common site of metastatic disease. The presence and extent of hepatic metastases are a major prognostic indicator. Although surgical resection is the accepted first-line therapy for colorectal liver metastasis, only 20 to $25 \%$ of patients are eligible for resection due to the extent and location of disease. This article discusses the current role of transarterial therapies in the treatment of colorectal liver metastases.
Objectives: Upon completion of this article, the reader will be able to discuss the role of chemoembolization and radioembolization in the treatment of colorectal liver metastases.

Accreditation: This activity has been planned and implemented in accordance with the Essential Areas and Policies of the Accreditation Council for Continuing Medical Education (ACCME) through the joint providership of Tufts University School of Medicine (TUSM) and Thieme Medical Publishers, New York. TUSM is accredited by the ACCME to provide continuing medical education for physicians.

Credit: Tufts University School of Medicine designates this journal-based CME activity for a maximum of $\mathbf{1} \boldsymbol{A M A}$ PRA Category 1 Credit $^{\mathrm{TM}}$. Physicians should claim only the credit commensurate with the extent of their participation in the activity.

Colorectal cancer is the third most common cancer in the United States, with the second highest mortality. The liver is the most common site of metastatic disease, with up to $50 \%$ of patients diagnosed with liver metastasis at the time of initial diagnosis. ${ }^{1}$ Further, $80 \%$ of patients develop liver metastases during their disease course. ${ }^{1}$ The presence and extent of liver metastases are a major prognostic indicator, with liver dysfunction secondary to metastatic disease being the cause of death in most patients. ${ }^{1,2}$

Surgical resection is accepted as the first-line treatment for colorectal liver metastasis (CRLM). Yet, at the time of diagnosis, only 20 to $25 \%$ of patients are eligible for resection of their CRLM. ${ }^{3,4}$ Current National Comprehensive Cancer Network (NCCN) guidelines recommend systemic chemotherapy with or without the addition of biologic therapies. ${ }^{5}$ Current systemic chemotherapies include 5-fluorouracil (5-FU)-based therapies consisting of 5-FU and oxaliplatin (FOLFOX), 5-FU and irinotecan (FOLFIRI), or capecitabine and oxaliplatin (CapOX). ${ }^{1,5}$ These therapies alone result in a response rates and overall survival (OS) of $40 \%$ and $57 \%$ at 15 to 20 months, respectively, yet OS nears $0 \%$ at 5 years. ${ }^{6-8}$ The addition of biologic therapies such as anti-vascular endothelial growth factor antibodies and antiepidermal growth factor receptor inhibitors has improved outcomes when used in conjunction with cytotoxic systemic chemotherapy. These combined regimens have increased OS to more than 24 months in several studies. ${ }^{9,10}$
Issue Theme Decision Making as a Growth Mechanism in Interventional Oncology; Guest Editor, Daniel B. Brown, MD, FSIR
Copyright (C) 2017 by Thieme Medical Publishers, Inc., 333 Seventh Avenue, New York, NY 10001, USA Tel: +1(212) 584-4662.
DOI https://doi.org/ 10.1055/s-0037-1601854. ISSN 0739-9529. 
Despite recent successes, these systemic therapies are difficult for patients to tolerate, with approximately one-third discontinuing the therapy before completing a full 12 cycles. ${ }^{11}$ Additional consideration of systemic therapies is needed in patients who may be downstaged from unresectable to resectable disease. Cytotoxic systemic therapies can induce chemotherapy-associated liver injury (CALI). For example, sinusoidal obstruction syndrome can result from prolonged treatment with oxaliplatin, particularly when used for more than 6 cycles. ${ }^{12}$ Steatohepatitis is associated with irinotecan administration, in addition to resulting in increased liver failure and death following hepatic resection. ${ }^{12}$

Adjuvant intra-arterial locoregional therapies (LRTs) can potentially mitigate some of the unwanted side effects of systemic therapy. LRTs are based on the concept of delivery of toxic substances directly to the tumor. Normal hepatocytes are predominately supplied by the portal vein, whereas tumor cells are supplied by the hepatic arteries. ${ }^{13}$ Unlike systemic chemotherapy, intra-arterial delivery of chemotherapy increases drug concentrations within the tumor and minimal circulating systemic drug, which results in fewer systemic toxicities. ${ }^{14}$ Current intra-arterial therapies include ${ }^{1}$ conventional transarterial chemoembolization (CTACE), ${ }^{2}$ transarterial chemoembolization with drug-eluting beads (DEB-TACE), and $^{3}$ transarterial radioembolization (TARE).

\section{CTACE}

The premise of chemoembolization is to combine local highdose chemotherapy and ischemia for enhanced tumor destruction, potentially in a synergistic manner. Conventional chemoembolization (CTACE) delivers chemotherapeutic agents emulsified with lipiodol, a poppy seed oil containing $38 \%$ iodine by weight. This is followed by the delivery of an embolic agent, often polyvinyl alcohol (PVA) or Gelfoam. Level I data have demonstrated the efficacy of CTACE in hepatocellular carcinoma (HCC). ${ }^{15,16}$ Because of the large survival benefit in HCC, several series have analyzed the role of CTACE in CRLM.

Lang and Brown reported 46 patients treated with CTACE between 1985 and 1991 using doxorubicin and lipiodol. ${ }^{17}$ The mean OS was 23 months versus 9 to 17 months with systemic therapy. Complications were observed in $33 \%$ (15/46) of patients and included suppressed hematopoiesis $(n=6)$, renal failure $(n=2)$, liver failure $(n=6)$, and diabetes $(n=2)$. Most of the patients experiencing suppressed hematopoiesis or liver failure underwent numerous sessions of chemoembolization or received additional systemic chemotherapy. This study demonstrated the feasibility and potential benefit of CTACE for patients with hepatic-dominant colorectal metastases.

A subsequent phase II trial evaluated chemoembolization with an emulsion of 5-FU, mitomycin C, and lipiodol followed by Gelfoam embolization in the salvage setting. ${ }^{18}$ Therefore, all patients had previously been treated with systemic chemotherapy. Radiologic responses were seen in $63 \%$ of cases, and $95 \%$ of patient experienced a decrease in carcinoembryonic antigen of more than $25 \%$. Median OS from date of first CTACE was 10 months. Longer median OS was seen in patients with more favorable performance status (24 months), metastatic disease confined to the liver (14 months), and serum alkaline phosphatase and lactate dehydrogenase less than three times normal (24 and 12 months, respectively). The most common toxicity was a postembolization syndrome (PES) including fever, right upper quadrant pain, nausea, and vomiting. Lethargy was noted for up to 6 weeks postembolization.

Albert et al presented a single institutional experience, wherein 245 cTACEs were performed in 121 patients with CRLM between 1992 and 2008. ${ }^{19}$ Patients were included if they had unresectable liver-dominant disease and had failed systemic chemotherapy. cTACE was performed with cisplatin, doxorubicin, mitomycin $\mathrm{C}$, and lipiodol mixture followed by PVA. Patients underwent a mean of two cTACE sessions. Performance status was preserved in $78 \%$ of patients with an Eastern Cooperative Oncology Group (ECOG) of 0 at the time of their first CTACE. Median OS was 33 months from initial diagnosis. Subgroup analysis demonstrated significantly better OS when CTACE was performed after first- or second-line chemotherapy as compared with in the salvage setting, after three to five lines of chemotherapy $(p=0.03$ ). Similar to prior reports, the most commonly experienced toxicity was a PES.

Vogl et al reported 463 patients treated with CTACE from 1999 to $2006 .{ }^{20}$ Several regimens were used, including mitomycin $\mathrm{C}$ alone, mitomycin $\mathrm{C}$ with gemcitabine, or mitomycin $C$ with irinotecan, depending on prior chemotherapy history. The indications for therapy included unresectable liver metastases without response to systemic therapy, disease progression, or intolerance to systemic therapy. Patients with extrahepatic disease or a Karnofsky status less than $70 \%$ were excluded. Median OS from the time of initial diagnosis was 38 months. No significant survival difference was observed between CTACE regimens $(p=0.534)$. As with prior studies, the most commonly experienced toxicities of the procedure were abdominal pain, nausea, and vomiting.

\section{DEB-TACE}

Drug-eluting bead (DEB) therapy consists of highly absorbent microspheres coated with high doses of chemotherapeutic agents which are then administered via the hepatic artery. The beads elute the drug over time, ${ }^{21,22}$ theoretically mitigating the systemic side effects experienced in CTACE. Several studies have evaluated the use of DEB loaded with irinotecan (DEBIRI) for CRLM.

Bower et al reported 55 patients who underwent 90 DEBIRI treatment sessions. All patients had received prior systemic chemotherapy. ${ }^{23}$ Eleven patients (20\%) demonstrated response with down staging or stable disease without extrahepatic disease progression allowing for hepatic resection $(n=6)$, radiofrequency ablation $(n=3)$, or a combination of resection and ablation $(n=2)$. Pathologic review of resected specimens demonstrated embolic beads near the tumor capsule with relative sparing of normal parenchyma. Histopathology demonstrated minimal portal inflammation 
and no evidence of chemotherapy-associated steatohepatitis or fibrosis.

Martin et al reported 55 patients who underwent 99 DEBIRI treatments between 2006 and 2008. ${ }^{24}$ All patients had received first-line systemic therapy with FOLFOX and bevacizumab, with many receiving second- and third-line therapy. Fifty percent of the patients had concurrent limited extrahepatic disease at the time of referral. In this salvage setting, median progression-free survival (PFS) was 11 months and median OS was 19 months from first DEBIRI.

Fiorentini et al randomized patients with unresectable CRLM to receive either DEBIRI $(n=35)$ or FOLFIRI $(n=35) .{ }^{25}$ Patients in the DEBIRI arm had prolonged median OS (22 vs. 15 months), greater likelihood of objective tumor response (68.6\% vs. $20 \%$ ), and sustained quality of life over systemic FOLFIRI ( 8 vs. 3 months, $p<0.001$ ). A major drawback of this study is the omission of oxaliplatin, bevacizumab, cetuximab, or panitumumab, as these agents were not standard of care at the time of the study. These agents when added to FOLFIRI demonstrate increased OS over FOLFIRI alone. ${ }^{26-28}$

A major drawback of DEBIRI has been the associated severe abdominal pain reported in $40 \%$ of patients. ${ }^{25}$ To overcome this, several protocols have been proposed to achieve appropriate pain control, including intravenous morphine and bolus lidocaine into the hepatic artery at the time of DEBIRI administration. ${ }^{29}$ More recently, paravertebral thoracic blocks with ultrasound guidance at the T6-T8 levels has been proposed for periprocedure pain control with side effects seen in less than $1 \%$ of patients. ${ }^{29}$

\section{Radioembolization}

TARE is the injection of micron-sized Yttrium-90 $\left({ }^{90} \mathrm{Y}\right)$ resin or glass particles into the hepatic artery. The particles result in the local delivery of high doses of $\beta$ radiation to the hepatic tumors. External beam radiation for CRLM is hampered by dose-limiting toxicities of the adjacent organs, respiratory and cardiac motion transmission to the liver, and the liver's radiosensitivity resulting in radiation-induced liver disease (RILD), a clinical syndrome of ascites, anicteric hepatomegaly, and elevated liver enzymes with exposures $>35 \mathrm{~Gy} .^{30}$ TARE helps deliver a more focused, high-dose radiation to the tumor with relative sparing of normal liver parenchyma and no toxicities to surrounding organs. TARE has been investigated both as a therapy in cases of metastatic disease refractory to chemotherapy and as part of first-line therapy.

Saxena et al reported 302 patients who underwent ${ }^{90} \mathrm{Y}$ for the treatment of unresectable, chemorefractory CRLM between 2006 and 2013. ${ }^{31}$ The median OS after TARE was 10.5 months. Complete response was seen in 2 patients (1\%), partial response seen in 111 (38\%), and stable disease in 96 (33\%). Factors associated with poor prognosis include extent of tumor and number of prior lines of chemotherapy. Clinical toxicities occurred in $38 \%$ of patients, most of which were mild and self-limited, including nausea/vomiting (26\%), abdominal pain (18\%), fatigue (17\%), and anorexia (8\%). However, more serious side effects including gastrointestinal ulceration and radiation-induced lung disease were reported in two patients.
Lewandowski et al reported on 214 patients treated with ${ }^{90} \mathrm{Y}$ for CRLM. ${ }^{32}$ Median OS was 43.0, 34.6, and 10.6 months from the date of diagnosis of primary cancer, hepatic metastases, and first ${ }^{90} \mathrm{Y}$, respectively. Survival was significantly longer in patients who received two or fewer cytotoxic drugs' regimens ( $p=0.0001)$ and patients who received no biologic agents ( $p=0.0001)$, supporting the use of ${ }^{90} Y$ earlier in the disease course. Clinical toxicities included fatigue, pain, nausea, vomiting, and fever. No gastrointestinal ulcerations, RILD, or pneumonitis was reported.

Despite growing evidence of improved outcomes with earlier implementation of ${ }^{90} \mathrm{Y}$ in the metastatic colorectal treatment algorithm, this has not been widely adopted. A review of 20 studies including 979 patients treated with TARE for refractory CRLM demonstrated that ${ }^{90} \mathrm{Y}$ is most often used in the salvage setting. Approximately one-third of the studies included patients with extrahepatic disease. This review reported a median OS of 12 months after ${ }^{90}$ Yand a median time to intrahepatic disease progression of 9 months. ${ }^{31}$

With growing interest in implementation of ${ }^{90} \mathrm{Y}$, several studies have investigated the use of concurrent systemic chemotherapy and TARE. Van Hazel et al presented a phase II randomized control trial of 21 patients comparing systemic 5-FU/leucovorin alone to patients first receiving a single injection of ${ }^{90} \mathrm{Y}$ microspheres. ${ }^{33}$ Patients receiving the combination therapy had a longer median OS (29.4 vs. 12.8 months, $p=0.02$ ) and time to disease progression (18.6 vs. 3.6 months). However, greater toxicities were seen in the combination arm, including one death due to neutropenic sepsis.

SIRFLOX was a phase III multicenter, international, randomized trial combining first-line chemotherapy with FOLFIRI (with bevacizumab at the discretion of the investigator) alone or in combination with ${ }^{90} \mathrm{Y}$ for liver-dominant or isolated CRLM in chemo-naïve patients. ${ }^{34} \mathrm{~A}$ total of 530 patients were randomized between 2006 and 2013. While PFS at any site was not significantly different between the two sites, hepatic PFS was significantly longer in those getting combination therapy (20.5 vs. 12.6 months, $p=0.002$ ). Follow-up data on OS are still being gathered.

\section{Patient Selection}

Assessment of patients being considered for intra-arterial liver-directed therapy includes evaluation of liver function, tumor size and distribution, involvement of the portal vein, hepatic arterial anatomy, comorbidities, and performance status. In the initial clinical evaluation of the patient, an understanding of their full oncologic history is important. While current data support ${ }^{90} Y$ as the initial LRT in the setting of CRLM, patients with prior radiation to the liver may not be candidates for the therapy. Additionally, some element of systemic chemotherapy-induced CALI may be present in patients who have been heavily treated with systemic therapy, even in the setting of relatively normal liver functions. ${ }^{12}$ Full understanding of underlying liver dysfunction is important to minimize the risk of hepatic failure following LRT.

While no absolute cut-offs exist, generally bilirubin less than $2 \mathrm{mg} / \mathrm{dL}$ represents the upper limit of what is acceptable to proceed with TACE or TARE. ${ }^{35}$ Early experience with TACE 
noted rates of hepatic insufficiency of 22.4 to $66.7 \% .^{36,37}$ This is often transient and reversible, particularly in patients with preserved hepatic function. ${ }^{38}$ Conversely, irreversible deterioration and acute liver failure is more often seen in patients with severe impairment in functional reserve. ${ }^{36}$ Radiation-induced liver failure after ${ }^{90} \mathrm{Y}$ is seen in 0 to $4 \%$ of patients, most often those with preexisting liver dysfunction and patients receiving single-session whole-liver radioembolization. ${ }^{39}$

Trends are also important to note: a sudden elevation of the patient's total bilirubin, even when the absolute value is less than 2, may represent worsening liver disease. In cases of borderline liver function, a very targeted TACE may be an approach to treat tumor(s) in a small localized distribution, while preserving the remaining liver.

Prior history of biliary intervention including percutaneous biliary drainage, stenting, sphincterotomy, or bilioenteric anastomosis increases the risk of biliary necrosis and abscess formation after TACE by as much as 800 -fold. ${ }^{40}$ Mezhir et al reported 14 abscesses among 971 patients after 2,045 TACEs. Of the 14 abscesses, 13 (93\%) occurred in patients with compromised sphincter of Oddi. ${ }^{41}$ This increased risk is attributed to bacterial colonization of the bile ducts followed by ischemic insult secondary to hepatic artery embolization. ${ }^{42}$ The risk may be decreased, but remains elevated despite aggressive antibiotic prophylaxis. ${ }^{43}$

In contrast, reports of abscess formation after TARE are rare and several small studies suggest a lower risk of abscess in patients with prior biliary intervention after TARE as compared with TACE. Cholapranee et al compared a cohort of 13 patients undergoing 24 TACEs to 16 patients undergoing 24 TAREs. All patients had compromise of their sphincter of Oddi due to prior surgical or endoscopic therapy. Thirteen of 13 (100\%) post-TACE patients developed an abscess; however, no patient in the TARE cohort developed abscesses. ${ }^{44}$

As demonstrated in numerous studies for both TACE and ${ }^{90} \mathrm{Y}$, functional status is a predictor of OS post liver-directed therapy. ${ }^{20,32}$ As such, patients should have an ECOG performance status of $\leq 2$. In patients where tolerability of postprocedure effects is of concern, ${ }^{90} \mathrm{Y}$ may be a better option when feasible. While a PES is seen in CTACE, DEBIRI, and ${ }^{90} \mathrm{Y}$, the syndrome reported for ${ }^{90} \mathrm{Y}$ is least severe with most patients discharged the day of the procedure. ${ }^{45}$

When evaluating the hepatic arterial anatomy, additional care should be considered in patients where TARE or lobar/ whole-liver therapy is planned. Nontarget embolization of ${ }^{90} \mathrm{Y}$ microspheres to the stomach or bowel can have devastating effects. ${ }^{46}$ Pretreatment angiography to evaluate for nontarget vessels such as an aberrant right gastric artery arising from the left hepatic artery is paramount to minimize the risk of gastrointestinal ulceration. ${ }^{46}$

At the completion of the angiogram, ${ }^{99 \mathrm{~m}} \mathrm{Tc}-\mathrm{MAA}$ in injected into the hepatic artery. The subsequent ${ }^{99 \mathrm{~m}} \mathrm{Tc}-\mathrm{MAA}$ scan allows for an estimate of radiation dose to the lung with a planned TARE. ${ }^{47}$ Early data in patients treated with ${ }^{90} \mathrm{Y}$ suggested a risk of radiation pneumonitis with doses of 30 $\mathrm{Gy} /$ treatment or a $50 \mathrm{~Gy}$ cumulative dose. Subsequent data presented by Salem et al suggested these doses are well tolerated. ${ }^{47}$ Importantly, a large amount of shunting from the liver to the lungs can result in nonlethal doses of radiation to the target hepatic tumor (i.e., the microspheres pass through the liver resulting in nontherapeutic hepatic radiation doses). For this reason, a high shunt fraction may be a reason to reevaluate the use of ${ }^{90} \mathrm{Y}$. In some cases, this shunt can be reduced with bland embolization or TACE prior to ${ }^{90} \mathrm{Y}$.

\section{Conclusion}

CRLM are common in patients with colorectal carcinoma, and less than a quarter of patients are eligible for surgical resection. Current first-line therapy for unresectable CRLM remains systemic chemotherapy; however, liver-directed therapies represent a favorable approach due to their targeted approach. Many series describing intra-arterial approaches for CRLM exist, but vary greatly in patient demographics and prior therapies, making exact comparison difficult. However, DEBIRI has been shown to improve OS compared with systemic chemotherapy. In addition, the SIRFLOX trial demonstrated longer hepatic PFS in patients receiving a combination of ${ }^{90} \mathrm{Y}$ and systemic chemotherapy versus chemotherapy alone. Although the best time to introduce LRT to systemic therapy is still unknown, many studies have demonstrated that LRT should be considered earlier in the treatment of CRLM. Further well-designed prospective and/or randomized studies are needed to better understand the role and timing of these liver-directed therapies.

\section{References}

1 Bhutiani N, Martin RCG II. Transarterial therapy for colorectal liver metastases. Surg Clin North Am 2016;96(2):369-391

2 Tsai MS, Su YH, Ho MC, et al. Clinicopathological features and prognosis in resectable synchronous and metachronous colorectal liver metastasis. Ann Surg Oncol 2007;14(2):786-794

3 Bentrem DJ, Dematteo RP, Blumgart LH. Surgical therapy for metastatic disease to the liver. Annu Rev Med 2005;56:139-156

4 LeGolvan MP, Resnick M. Pathobiology of colorectal cancer hepatic metastases with an emphasis on prognostic factors. J Surg Oncol 2010;102(8):898-908

5 NCCN Guidelines Version 2. 2015 Colon Cancer, COL-7. Available at: https://www.scribd.com/document/264433721/NCCN-Colon. Accessed on April 5, 2017

6 Schwarz RE, Berlin JD, Lenz HJ, Nordlinger B, Rubbia-Brandt L, Choti MA. Systemic cytotoxic and biological therapies of colorectal liver metastases: expert consensus statement. HPB (Oxford) 2013;15(2):106-115

7 Goldberg RM, Sargent DJ, Morton RF, et al. Randomized controlled trial of reduced-dose bolus fluorouracil plus leucovorin and irinotecan or infused fluorouracil plus leucovorin and oxaliplatin in patients with previously untreated metastatic colorectal cancer: a North American Intergroup Trial. J Clin Oncol 2006;24(21): 3347-3353

8 Thirion P, Michiels S, Pignon JP, et al; Meta-Analysis Group in Cancer. Modulation of fluorouracil by leucovorin in patients with advanced colorectal cancer: an updated meta-analysis. J Clin Oncol 2004;22(18):3766-3775

9 Foubert F, Matysiak-Budnik T, Touchefeu Y. Options for metastatic colorectal cancer beyond the second line of treatment. Dig Liver Dis 2014;46(2):105-112

10 Zhao J, Du CZ, Sun YS, Gu J. Patterns and prognosis of locally recurrent rectal cancer following multidisciplinary treatment. World J Gastroenterol 2012;18(47):7015-7020 
11 Ji JH, Park SH, Lee J, et al. Prospective phase II study of neoadjuvant FOLFOX6 plus cetuximab in patients with colorectal cancer and unresectable liver-only metastasis. Cancer Chemother Pharmacol 2013;72(1):223-230

12 Abdalla EK, Vauthey JN. Chemotherapy prior to hepatic resection for colorectal liver metastases: helpful until harmful? Dig Surg 2008;25(6):421-429

13 Breedis C, Young G. The blood supply of neoplasms in the liver. Am J Pathol 1954;30(5):969-977

14 Collins JM. Pharmacologic rationale for regional drug delivery. J Clin Oncol 1984;2(5):498-504

15 Llovet JM, Real MI, Montaña X, et al; Barcelona Liver Cancer Group. Arterial embolisation or chemoembolisation versus symptomatic treatment in patients with unresectable hepatocellular carcinoma: a randomised controlled trial. Lancet 2002;359(9319):1734-1739

16 Lo CM, Ngan H, Tso WK, et al. Randomized controlled trial of transarterial lipiodol chemoembolization for unresectable hepatocellular carcinoma. Hepatology 2002;35(5):1164-1171

17 Lang EK, Brown CL Jr. Colorectal metastases to the liver: selective chemoembolization. Radiology 1993;189(2):417-422

18 Tellez C, Benson AB III, Lyster MT, et al. Phase II trial of chemoembolization for the treatment of metastatic colorectal carcinoma to the liver and review of the literature. Cancer 1998;82(7): 1250-1259

19 Albert M, Kiefer MV, Sun W, et al. Chemoembolization of colorectal liver metastases with cisplatin, doxorubicin, mitomycin C, ethiodol, and polyvinyl alcohol. Cancer 2011;117(2):343-352

20 Vogl TJ, Gruber T, Balzer JO, Eichler K, Hammerstingl R, Zangos S. Repeated transarterial chemoembolization in the treatment of liver metastases of colorectal cancer: prospective study. Radiology 2009;250(1):281-289

21 Lewis AL, Gonzalez MV, Lloyd AW, et al. DC bead: in vitro characterization of a drug-delivery device for transarterial chemoembolization. J Vasc Interv Radiol 2006;17(2 Pt 1):335-342

22 Taylor RR, Tang Y, Gonzalez MV, Stratford PW, Lewis AL. Irinotecan drug eluting beads for use in chemoembolization: in vitro and in vivo evaluation of drug release properties. Eur J Pharm Sci 2007;30(1):7-14

23 Bower M, Metzger T, Robbins K, et al. Surgical downstaging and neo-adjuvant therapy in metastatic colorectal carcinoma with irinotecan drug-eluting beads: a multi-institutional study. HPB (Oxford) 2010;12(1):31-36

24 Martin RCG, Joshi J, Robbins K, et al. Hepatic intra-arterial injection of drug-eluting bead, irinotecan (DEBIRI) in unresectable colorectal liver metastases refractory to systemic chemotherapy: results of multi-institutional study. Ann Surg Oncol 2011;18(1): 192-198

25 Fiorentini G, Aliberti C, Tilli M, et al. Intra-arterial infusion of irinotecan-loaded drug-eluting beads (DEBIRI) versus intravenous therapy (FOLFIRI) for hepatic metastases from colorectal cancer: final results of a phase III study. Anticancer Res 2012; 32(4):1387-1395

26 Cunningham D, Humblet Y, Siena S, et al. Cetuximab monotherapy and cetuximab plus irinotecan in irinotecan-refractory metastatic colorectal cancer. N Engl J Med 2004;351(4):337-345

27 Hurwitz H, Fehrenbacher L, Novotny W, et al. Bevacizumab plus irinotecan, fluorouracil, and leucovorin for metastatic colorectal cancer. N Engl J Med 2004;350(23):2335-2342

28 Chen HX, Mooney M, Boron M, et al. Phase II multicenter trial of bevacizumab plus fluorouracil and leucovorin in patients with advanced refractory colorectal cancer: an NCI Treatment Referral Center Trial TRC-0301. J Clin Oncol 2006;24(21):3354-3360

29 Marqués A, García R, Gómez F, Giammaria F. Ultrasound-guided paravertebral block for management of abdominal pain after transarterial embolization using drug-eluting beads loaded with irinotecan. J Vasc Interv Radiol 2013;24(9):1416-1417
30 Geschwind JF, Salem R, Carr BI, et al. Yttrium-90 microspheres for the treatment of hepatocellular carcinoma. Gastroenterology 2004;127(5, Suppl 1):S194-S205

31 Saxena A, Bester L, Shan L, et al. A systematic review on the safety and efficacy of yttrium-90 radioembolization for unresectable, chemorefractory colorectal cancer liver metastases. J Cancer Res Clin Oncol 2014;140:537-547

32 Lewandowski RJ, Memon K, Mulcahy MF, et al. Twelve-year experience of radioembolization for colorectal hepatic metastases in 214 patients: survival by era and chemotherapy. Eur J Nucl Med Mol Imaging 2014;41(10):1861-1869

33 Van Hazel G, Blackwell A, Anderson J, et al. Randomised phase 2 trial of SIR-Spheres plus fluorouracil/leucovorin chemotherapy versus fluorouracil/leucovorin chemotherapy alone in advanced colorectal cancer. J Surg Oncol 2004;88(2):78-85

34 van Hazel GA, Heinemann V, Sharma NK, et al. SIRFLOX: randomized phase iii trial comparing first-line mFOLFOX6 (plus or minus bevacizumab) versus mFOLFOX6 (plus or minus bevacizumab) plus selective internal radiation therapy in patients with metastatic colorectal cancer. J Clin Oncol 2016;34(15):1723-1731

35 Georgiades CS, Hong K, Geschwind JF. Pre- and postoperative clinical care of patients undergoing interventional oncology procedures: a comprehensive approach to preventing and mitigating complications. Tech Vasc Interv Radiol 2006;9(3):113-124

36 Chung JW, Park JH, Han JK, et al. Hepatic tumors: predisposing factors for complications of transcatheter oily chemoembolization. Radiology 1996;198(1):33-40

37 Buijs M, Vossen JA, Frangakis C, et al. Nonresectable hepatocellular carcinoma: long-term toxicity in patients treated with transarterial chemoembolization-single-center experience. Radiology 2008;249(1):346-354

38 Yamada R, Sato M, Kawabata M, Nakatsuka H, Nakamura K, Takashima S. Hepatic artery embolization in 120 patients with unresectable hepatoma. Radiology 1983;148(2):397-401

39 Sangro B, Gil-Alzugaray B, Rodriguez J, et al. Liver disease induced by radioembolization of liver tumors: description and possible risk factors. Cancer 2008;112(7):1538-1546

40 Kim W, Clark TW, Baum RA, Soulen MC. Risk factors for liver abscess formation after hepatic chemoembolization. J Vasc Interv Radiol 2001;12(8):965-968

41 Mezhir JJ, Fong Y, Fleischer D, et al. Pyogenic abscess after hepatic artery embolization: a rare but potentially lethal complication. J Vasc Interv Radiol 2011;22(2):177-182

42 Kim HK, Chung YH, Song BC, et al. Ischemic bile duct injury as a serious complication after transarterial chemoembolization in patients with hepatocellular carcinoma. J Clin Gastroenterol 2001;32(5):423-427

43 Patel S, Tuite CM, Mondschein JI, Soulen MC. Effectiveness of an aggressive antibiotic regimen for chemoembolization in patients with previous biliary intervention. J Vasc Interv Radiol 2006; 17(12):1931-1934

44 Cholapranee A, van Houten D, Deitrick G, et al. Risk of liver abscess formation in patients with prior biliary intervention following yttrium-90 radioembolization. Cardiovasc Intervent Radiol 2015; 38(2):397-400

45 Salem R, Lewandowski RJ, Atassi B, et al. Treatment of unresectable hepatocellular carcinoma with use of $90 \mathrm{Y}$ microspheres (TheraSphere): safety, tumor response, and survival. J Vasc Interv Radiol 2005;16(12):1627-1639

46 Riaz A, Lewandowski RJ, Kulik LM, et al. Complications following radioembolization with yttrium-90 microspheres: a comprehensive literature review.J Vasc Interv Radiol 2009;20(9):1121-1130, quiz 1131

47 Salem R, Parikh P, Atassi B, et al. Incidence of radiation pneumonitis after hepatic intra-arterial radiotherapy with yttrium-90 microspheres assuming uniform lung distribution. Am J Clin Oncol 2008;31(5):431-438 